\title{
Flame retardancy and kinetic behavior of ammonium polyphosphate-treated unsaturated polyester/phenolic interpenetrating polymer network.
}

\begin{abstract}
In this study, the flammability and kinetic behavior of flame retardant unsaturated polyester (UP)/phenolic resin were investigated. The flame retardant ammonium polyphosphate (APP) was used in this research to improve the flame resistance of a UP/phenolic resin interpenetrating polymer network (IPN). The flame resistance of UP improved from none to V-0 classification by adding phenolic resin and APP. Kinetic behavior study of UP, UP/phenolic, and APP-filled UP/phenolic IPN was carried out by the Borchardt and Daniels method. The results indicated that modification of flammable UP resin markedly improved the total heat release volume of UP and the flame retardancy of the IPN network structure was also enhanced.
\end{abstract}

Keyword: Ammonium polyphosphate; Flammability; Kinetic; Phenolic; Unsaturated polyester. 